Case Report

\title{
Undifferentiated Pleomorphic Sarcoma Presenting as Abdominal Pain with a Pulsatile Mass
}

\author{
Arash Moradi, ${ }^{1}$ Abolfazl Afsharfard, ${ }^{1}$ and Khashayar Atqiaee ${ }^{2}$ \\ ${ }^{1}$ Department of General and Vascular Surgery, Shohada-e-Tajrish Medical Center, \\ Shahid Beheshti University of Medical Sciences, Tehran, Iran \\ ${ }^{2}$ Department of Pediatric Surgery, Mofid Children's Hospital, Shahid Beheshti University of Medical Sciences, Tehran, Iran \\ Correspondence should be addressed to Arash Moradi; dr.arash-moradi@hotmail.com
}

Received 6 February 2016; Accepted 5 July 2016

Academic Editor: Jochen Tüttenberg

Copyright (C) 2016 Arash Moradi et al. This is an open access article distributed under the Creative Commons Attribution License, which permits unrestricted use, distribution, and reproduction in any medium, provided the original work is properly cited.

\begin{abstract}
Malignant fibrous histiocytoma (MFH) is a rare tumor that mostly involves adults aged 50 to 70 . The most common anatomic location is the lower extremities. MFH of the retroperitoneum usually manifests late in its course and may be initially mistaken with other more common diagnosis. Here, the authors describe a 60-year-old man that was brought to the emergency department with a chief complaint of periumbilical abdominal pain. Our patient presented with symptoms consistent with a symptomatic aortic aneurysm, but a mass was encountered during surgery. In such circumstances the diagnosis of malignant sarcoma must be kept in mind and attempts at full resection with tumor-free margins are necessary.
\end{abstract}

\section{Background}

Malignant fibrous histiocytoma (MFH) was first described in 1961 by Kauffman and Stout as a histiocytic tumor with storiform growth in children [1]. Its malignant nature combined with an uncertainty regarding its origin and ability to involve both bone and soft tissue has given it the characteristics of a mysterious tumor, which is now accepted as a formal diagnosis of "undifferentiated pleomorphic sarcoma not otherwise specified" [2]. Various subtypes of MFH have been recognized including storiform-pleomorphic, myxoid, giant cell, and inflammatory [3]. Of these the storiformpleomorphic subtype is the most common accounting for around $70 \%$ of the cases.

This rare tumor mostly involves adults aged 50 to 70 , with a slight tendency towards male gender. The most common anatomic location is the lower extremities and the most common presentation is a growing painless soft tissue lump. Here we present the case of a 60 -year-old man who came to the emergency department with a complaint of abdominal pain.

\section{Case Report}

A 60-year-old man was brought to the emergency department with a chief complaint of periumbilical abdominal pain. He suffered from abdominal pain for two weeks but it was significantly increased in the three days prior to admission. The pain radiated to the back and no accompanying signs or symptoms were present. It was described as a constant pain without any alleviating or exacerbating factors. The patient was a heavy smoker without any history of previous surgery or drug use.

The patient showed stable vital signs and a nuisance general appearance. No objective signs of weight loss or cachexia were observed. The physical exam was normal in the head and neck, chest, and extremities. In the abdomen a pulsatile mass was palpated in the periumbilical region. Routine laboratory tests revealed mild normocytic normochromic anemia, normal ESR and CRP, and liver function and diagnostic tests within normal limits.

Due to the pulsatile nature of the mass a CT angiography of the abdomen was ordered to look for vascular pathology. The tomography revealed a 5-by- $4 \mathrm{~cm}$ pseudoaneurysm with 
hematoma formation in the infrarenal aorta alongside diffuse dilation of the infrarenal inferior vena cava with extension into both common iliac veins (Figure 1), and the patient was prepped for surgical repair.

During surgery a pulsatile mass 20 by $20 \mathrm{~cm}$ in diameter with surrounding hematoma was observed in left zones 1 and 2. Upon further investigation a huge retroperitoneal mass 11 by 8 by $6 \mathrm{~cm}$ in diameter with apparent local invasion to the aorta and the inferior vena cava was found. En bloc resection was attempted along with involved vascular segments. The abdominal aorta was reconstructed using $16 \mathrm{~mm}$-by- $8 \mathrm{~mm}$ Dacron bifurcated graft, while the IVC was ligated below the renal veins but perhaps because of chronic process of disease no lower extremity edema happened and the patient was discharged after a week.

Pathologic evaluation of the specimen showed an undifferentiated pleomorphic sarcoma (a.k.a. malignant fibrous histiocytoma) with a histologic grade according to French Federation of Cancer Centers Sarcoma Group III (tumor differentiation: III, mitotic rate $>20 / \mathrm{HPF}$ : III, and tumor necrosis $<50 \%$ : I) (Figure 2). There was no evidence of lymphvascular or perineural invasion. Aneurysmal wall resection of the abdominal aorta with blood clot and atherosclerosis was also reported. The specimen was also positive for vimentin (patchy areas), SMA (small portions), and CD68 (most parts).

\section{Discussion}

Malignant fibrous histiocytoma is a sarcoma of mesenchymal origin affecting soft tissues of the body, particularly the extremities and retroperitoneum, yet it has been reported in almost all parts of the body [4-7]. The term malignant fibrous histiocytoma is believed to be a misnomer since the precise origin of MFH cells has been disputed and the concept of fibrohistiocytic differentiation has been challenged [8]. For this, the WHO has defined the tumor and its subtypes under undifferentiated pleomorphic sarcoma (UPS) not otherwise specified (NOS). Most undifferentiated high-grade pleomorphic sarcomas and undifferentiated pleomorphic sarcomas with giant cells occur in the deep soft tissues of the extremities while undifferentiated pleomorphic sarcoma with prominent inflammation is most commonly seen in the retroperitoneum [2].

Retroperitoneal and intra-abdominal tumors may present with constitutional symptoms, including fever, malaise, and weight loss $[9,10]$. The tumor is often large at presentation since it usually goes unnoticed for a long time and may cause displacement of the bowel, kidney, ureter, and/or bladder. In our patient the tumor had displaced an abdominal aortic aneurysm anteriorly thus creating the unique clinical presentation of abdominal pain and pulsatile mass.

Although the best imaging modality for evaluation of the tumor is Magnetic Resonance Imaging (MRI), for intraabdominal masses usually this is not the initial step. Generally, retroperitoneal undifferentiated pleomorphic sarcomas manifest as heterogeneous masses with areas of hemorrhage

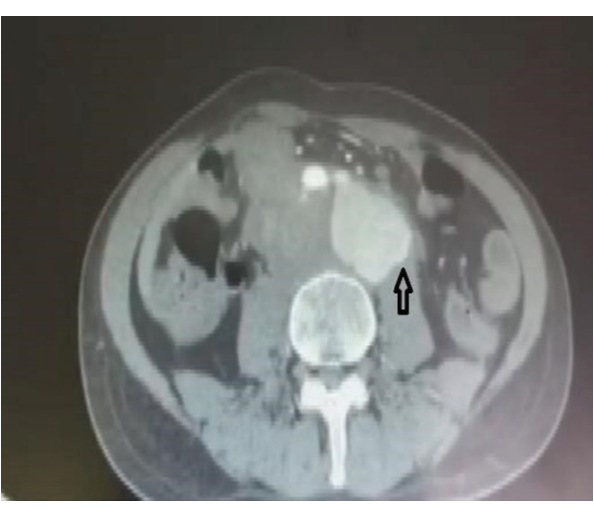

FIGURE 1: Large retroperitoneal mass.

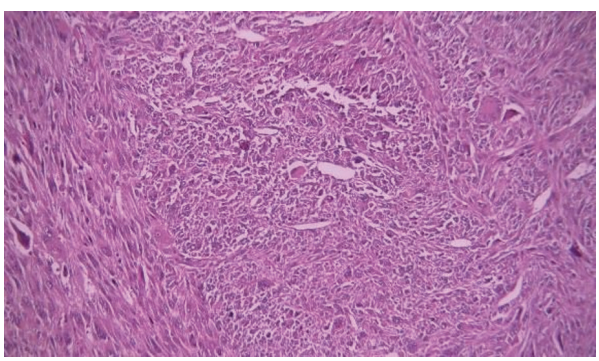

FIgURE 2: Hematoxylin and eosin stained section at 400x magnification demonstrating highly pleomorphic cells with prominent nucleoli in a storiform pattern.

and necrosis and occasionally focal or diffuse coarse calcifications. While invasion of the abdominal musculature is a wellrecognized phenomenon, the renal veins, or inferior vena cava, are not invaded $[11,12]$. In our patient too despite the initial impression of vascular invasion in the operating room, pathologic reports confirmed that the vascular invasion had not occurred.

One must bear in mind that the pathological diagnosis of MFH/UPS is a diagnosis of exclusion. In order to rule out a pleomorphic nonmesenchymal neoplasm resembling a pleomorphic sarcoma, pleomorphic sarcoma as a result of dedifferentiation, and pleomorphic sarcoma with a specific line of differentiation, a combination of sampling and immunohistochemistry should be used.

Microscopically, UPS has a highly variable morphologic pattern and shows storiform to haphazardly arranged pleomorphic areas. In immunohistochemistry they show features of fibroblasts/myofibroblasts [8].

The current treatment of choice for primary MFH/UPS is wide surgical resection aiming at tumor-free margins. While adjuvant radiotherapy has become an integral part of treatment, the role of chemotherapy is still controversial in these tumors [13-15].

\section{Conclusion}

MFH/UPS of the retroperitoneum usually manifests late in its course and may be initially mistaken with other more 
common diagnoses. Our patient presented with symptoms consistent with a symptomatic aortic aneurysm, but a mass was encountered during surgery. In such circumstances the diagnosis of malignant sarcoma must be kept in mind and attempts at full resection with tumor-free margins are necessary.

\section{Competing Interests}

The authors declare that they have no competing interests.

\section{References}

[1] S. L. Kauffman and A. P. Stout, "Histiocytic tumors (fibrous xanthoma and histiocytoma) in children," Cancer, vol. 14, pp. 469-482, 1961.

[2] C. Fetcher, K. Unni, and F. Mertens, World Health Organization Classification of Tumors: Pathology and Genetics of Soft Tissue and Bone, IARC Press, Lyon, France, 2002.

[3] M. D. Murphey, "World Health Organization classification of bone and soft tissue tumors: modifications and implications for radiologists," Seminars in Musculoskeletal Radiology, vol. 11, no. 3, pp. 201-214, 2007.

[4] D. Sadri and I. Yazdi, "Postradiation malignant fibrous histiocytoma of the maxillary sinus," Archives of Iranian Medicine, vol. 10, no. 3, pp. 393-396, 2007.

[5] N. Fujimura, Y. Sugita, M. Hirohata et al., "Primary intracerebral malignant fibrous histiocytoma in a child," Pediatric Neurosurgery, vol. 37, no. 5, pp. 271-274, 2002.

[6] C. Kotan, M. Kosem, S. Alici, M. Ilhan, I. Tuncer, and M. Harman, "Primary malignant fibrous histiocytoma of the small intestine presenting as an intussuSception: Report of a case," Surgery Today, vol. 32, no. 12, pp. 1091-1095, 2002.

[7] S. Gruttadauria, C. Doria, M. I. Minervini et al., "Malignant fibrous histiocytoma of the gallbladder: case report and review of the literature," The American Surgeon, vol. 67, no. 7, pp. 714717, 2001.

[8] J. R. Goldblum, "An approach to pleomorphic sarcomas: can we subclassify, and does it matter?" Modern Pathology, vol. 27, no. 1, pp. S39-S46, 2014.

[9] N. A. Qureshi, M. T. Hallissey, J. W. Fielding, and D. Gourevitch, "Primary intra-abdominal malignant fibrous histiocytoma presenting as pyrexia of unknown origin-report of a case with review of literature," International Seminars in Surgical Oncology, vol. 3, article 15, 2006.

[10] M. M. Gupta, N. Bahri, P. Watal et al., "Retroperitoneal cystic malignant fibrous histiocytoma mimicking a psoas abscess," Iranian Journal of Radiology, vol. 12, no. 3, Article ID e17507, 2015.

[11] N. Fang, Y.-L. Wang, L. Zeng, Z.-J. Wu, and X.-J. Cui, "Metastatic malignant fibrous histiocytoma in the stomach: imaging with 18F-FDG PET/CT,' Clinical Nuclear Medicine, vol. 41, no. 2, pp. e123-e124, 2016.

[12] W. Zheng, J. Chen, J. Liu, C. Zuo, and Z. Zhou, "FDG PET/CT findings of malignant fibrous histiocytoma of the stomach," Clinical Nuclear Medicine, vol. 40, no. 5, pp. 413-414, 2015.

[13] R. D. Lindberg, R. G. Martin, M. M. Romsdahl, and H. T. Barkley Jr., "Conservative surgery and postoperative radiotherapy in 300 adults with soft-tissue sarcomas," Cancer, vol. 47, no. 10, pp. 2391-2397, 1981.
[14] G. K. Zagars, J. R. Mullen, and A. Pollack, "Malignant fibrous histiocytoma: outcome and prognostic factors following conservation surgery and radiotherapy," International Journal of Radiation Oncology Biology Physics, vol. 34, no. 5, pp. 983-994, 1996.

[15] Sarcoma Meta-Analysis Collaboration, "Adjuvant chemotherapy for localised resectable soft-tissue sarcoma of adults: metaanalysis of individual data," The Lancet, vol. 350, no. 9092, pp. 1647-1654, 1997. 


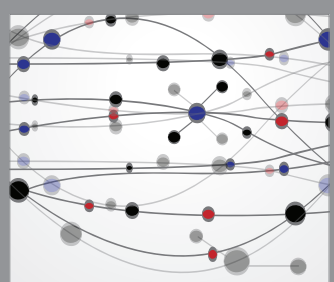

The Scientific World Journal
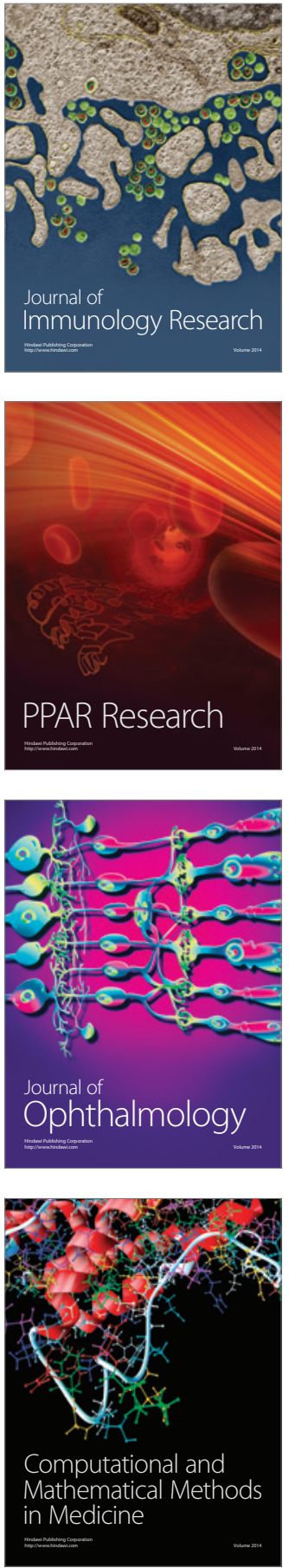

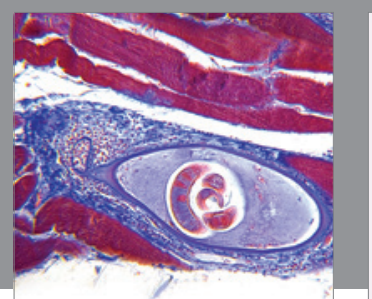

Gastroenterology Research and Practice

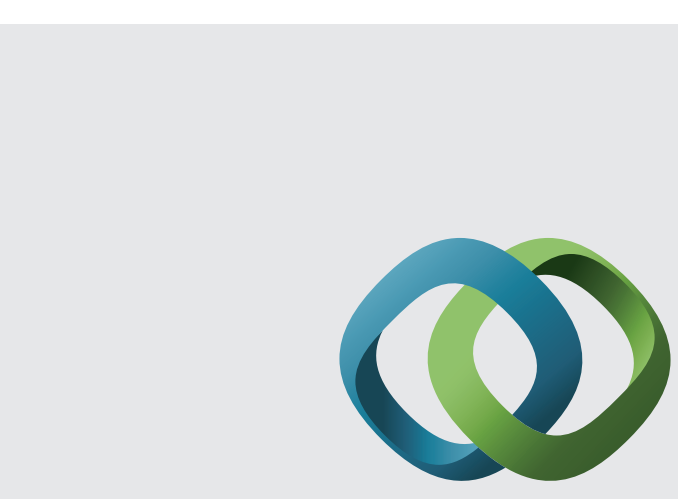

\section{Hindawi}

Submit your manuscripts at

http://www.hindawi.com
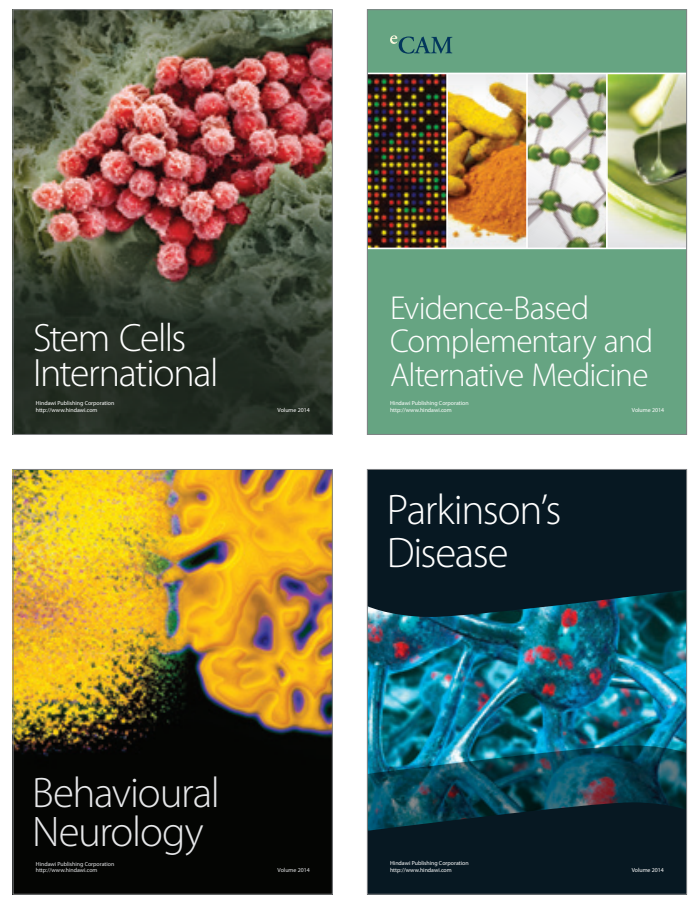
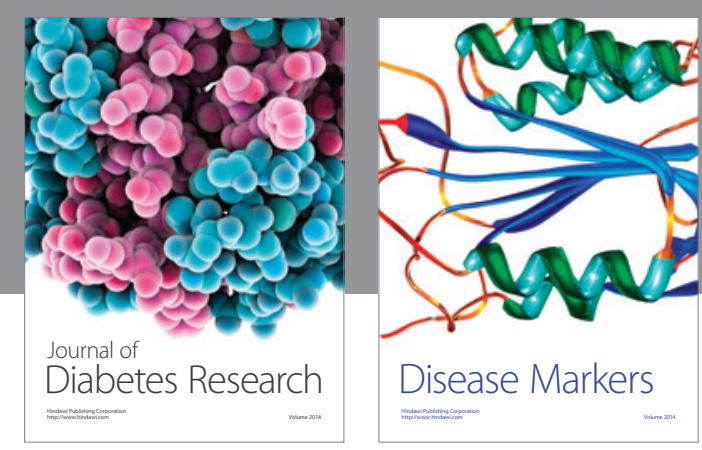

Disease Markers
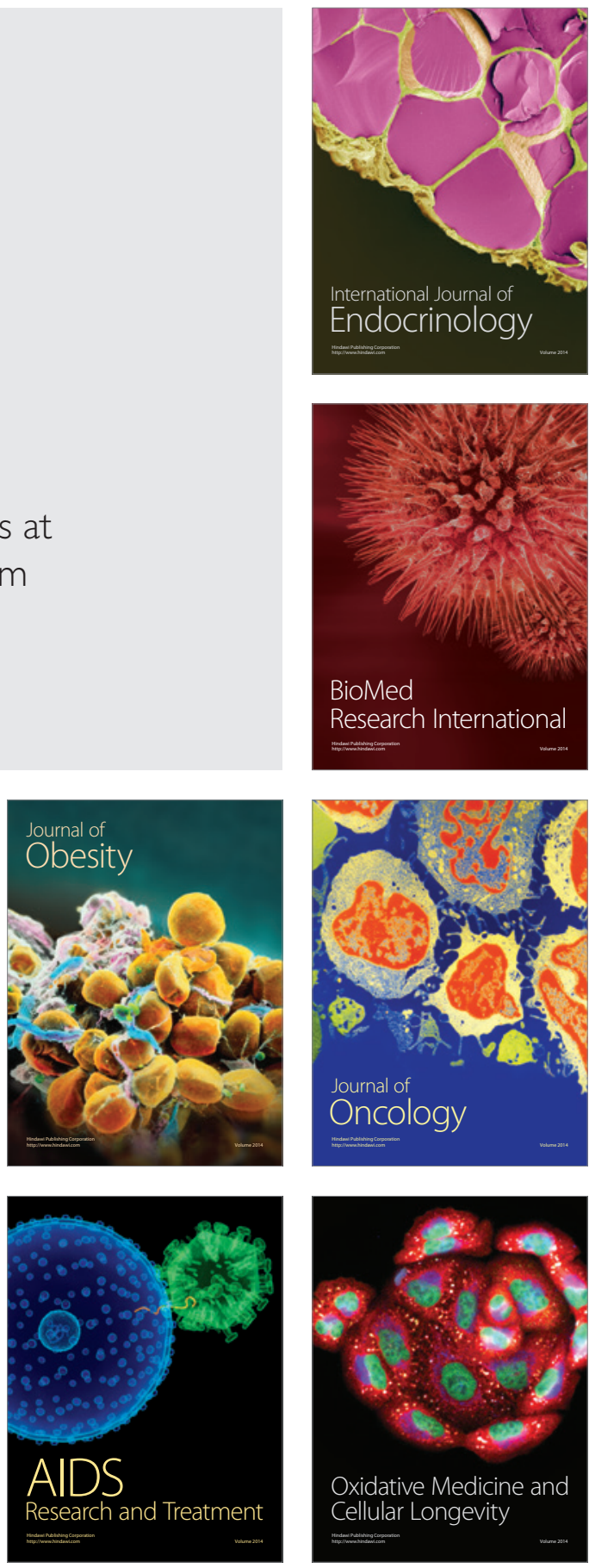